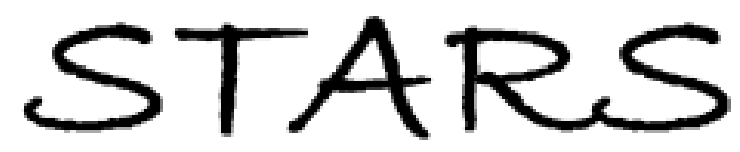

University of Central Florida

STARS

$1-1-2008$

\title{
Classical size effect in oxide-encapsulated Cu thin films: Impact of grain boundaries versus surfaces on resistivity
}

Tik Sun

University of Central Florida

Bo Yao

University of Central Florida

Andrew P. Warren

University of Central Florida

Vineet Kumar

Scott Roberts

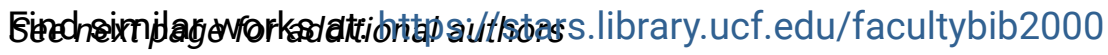

University of Central Florida Libraries http://library.ucf.edu

This Article is brought to you for free and open access by the Faculty Bibliography at STARS. It has been accepted for inclusion in Faculty Bibliography 2000s by an authorized administrator of STARS. For more information, please contactSTARS@ucf.edu.

\section{Recommended Citation}

Sun, Tik; Yao, Bo; Warren, Andrew P.; Kumar, Vineet; Roberts, Scott; Barmak, Katayun; and Coffey, Kevin R., "Classical size effect in oxide-encapsulated Cu thin films: Impact of grain boundaries versus surfaces on resistivity" (2008). Faculty Bibliography 2000s. 1033.

https://stars.library.ucf.edu/facultybib2000/1033

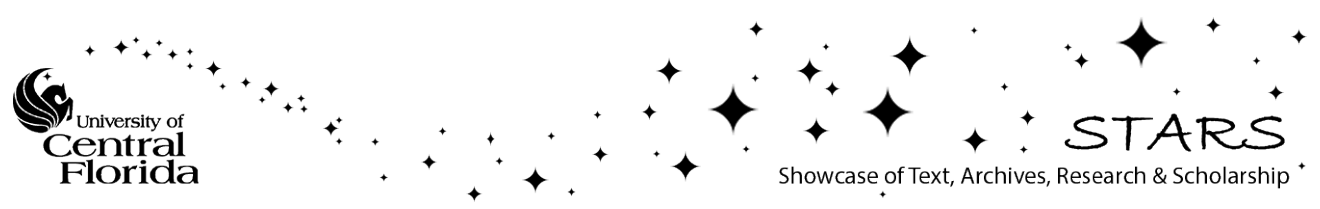


Authors

Tik Sun, Bo Yao, Andrew P. Warren, Vineet Kumar, Scott Roberts, Katayun Barmak, and Kevin R. Coffey 


\section{Classical size effect in oxide-encapsulated Cu thin films: Impact of grain boundaries versus surfaces on resistivity}

Tik Sun, Bo Yao, Andrew P. Warren, Vineet Kumar, Scott Roberts, Katayun Barmak, and Kevin R. Coffey

Citation: Journal of Vacuum Science \& Technology A 26, 605 (2008); doi: 10.1116/1.2938395

View online: https://doi.org/10.1116/1.2938395

View Table of Contents: https://avs.scitation.org/toc/jva/26/4

Published by the American Vacuum Society

\section{ARTICLES YOU MAY BE INTERESTED IN}

Surface and grain boundary scattering in nanometric Cu thin films: A quantitative analysis including twin boundaries

Journal of Vacuum Science \& Technology A 32, 061503 (2014); https://doi.org/10.1116/1.4894453

Electron mean free path in elemental metals

Journal of Applied Physics 119, 085101 (2016); https://doi.org/10.1063/1.4942216

Influence of surface and grain-boundary scattering on the resistivity of copper in reduced dimensions Applied Physics Letters 84, 2838 (2004); https://doi.org/10.1063/1.1703844

Alteration of $\mathrm{Cu}$ conductivity in the size effect regime Journal of Vacuum Science \& Technology B: Microelectronics and Nanometer Structures Processing, Measurement, and Phenomena 22, 240 (2004); https://doi.org/10.1116/1.1642639

Influence of phonon, geometry, impurity, and grain size on Copper line resistivity Applied Physics Letters 89, 113124 (2006); https://doi.org/10.1063/1.2355435

Size effects and charge transport in metals: Quantum theory of the resistivity of nanometric metallic structures arising from electron scattering by grain boundaries and by rough surfaces

Applied Physics Reviews 4, 011102 (2017); https://doi.org/10.1063/1.4974032

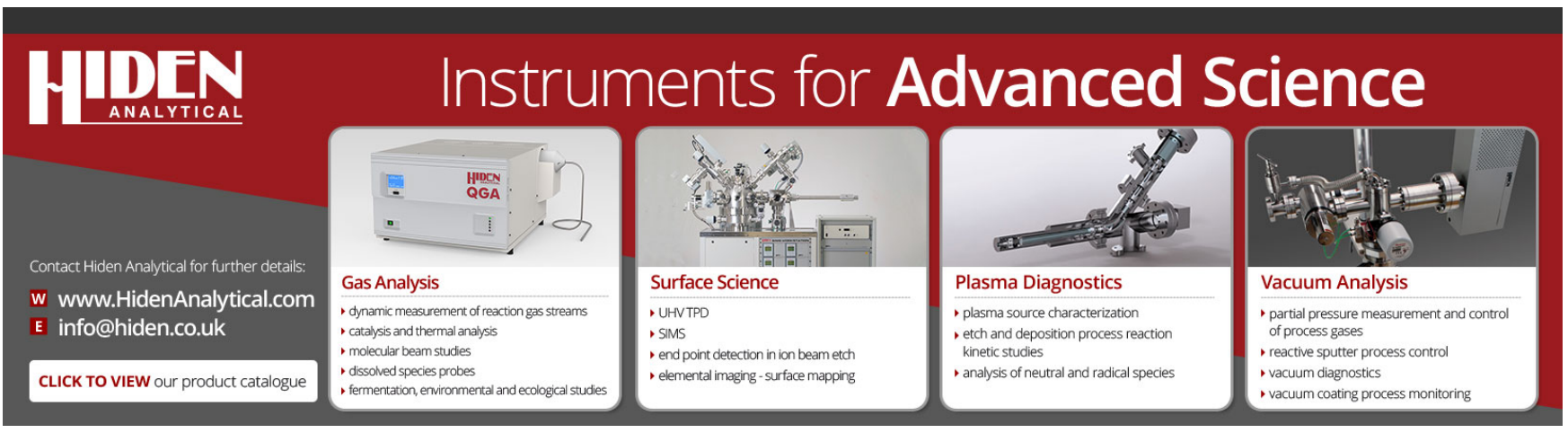




\title{
Classical size effect in oxide-encapsulated Cu thin films: Impact of grain boundaries versus surfaces on resistivity
}

\author{
Tik Sun, ${ }^{\text {a) }}$ Bo Yao, and Andrew P. Warren \\ Advanced Materials Processing and Analysis Center, University of Central Florida, 4000 Central Florida \\ Boulevard, Orlando, Florida 32816, USA
}

Vineet Kumar, Scott Roberts, and Katayun Barmak

Department of Materials Science and Engineering, Carnegie Mellon University, 5000 Forbes Avenue, Pittsburgh, Pennsylvania 15213, USA

Kevin R. Coffey ${ }^{\text {b) }}$

Advanced Materials Processing and Analysis Center, University of Central Florida, 4000 Central Florida Boulevard, Orlando, Florida 32816, USA

(Received 8 August 2007; accepted 5 May 2008; published 17 June 2008)

\begin{abstract}
A methodology is developed to independently evaluate surface and grain boundary scattering in silicon dioxide-encapsulated, polycrystalline $\mathrm{Cu}$ thin films. The room-temperature film resistivity for samples with film thicknesses in the range of 27 to $165 \mathrm{~nm}$ and different grain sizes (determined from approximately 400 to 1500 grains per sample) is compared to existing and empirical models of surface and grain boundary scattering. For the combined effects of surface and grain boundary scattering, the surface specularity parameter $p$ is $0.6 \pm 0.2$ and the grain boundary reflectivity coefficient $R$ is $0.45 \pm 0.03$. It is thereby shown that the resistivity contribution from grain boundary scattering is significantly greater than that of surface scattering for $\mathrm{Cu}$ thin films having $\mathrm{Cu} / \mathrm{SiO}_{2}$ surfaces and grain sizes similar to film thickness. (C) 2008 American Vacuum Society.
\end{abstract}

[DOI: $10.1116 / 1.2938395]$

\section{INTRODUCTION}

The classical size effect, wherein conductors with dimensions of the order of the mean free path of electrons exhibit higher resistivities, is illustrated in our data of Fig. 1, and was first noted more than 100 years ago. ${ }^{1}$ The importance of this effect to polycrystalline metal interconnects in silicon technology was first identified in $1998,{ }^{2}$ and subsequently several efforts were made to independently assess the grain boundary $^{3}$ and surface ${ }^{4}$ scattering contributions to resistivity. ${ }^{5-7}$ However, such an assessment is very challenging, requiring not only the preparation of suitably small conductors having independent variation of the two relevant length scales (the sample critical dimension and the grain size), but also independent, experimental quantification of these two length scales. ${ }^{8}$ Since for most work to date the sample grain size has been either assumed equal to conductor dimension or measured for only a small number of grains, the quantification of the classical size effect still suffers from an uncertainty in the relative contributions of surface and grain boundary scattering. ${ }^{7-9}$

This report describes a study where $\mathrm{SiO}_{2}$-encapsulated $\mathrm{Cu}$ thin film conductors ranging in thickness from approximately 30 to $160 \mathrm{~nm}$ are prepared at cryogenic deposition temperatures and annealed to provide an independent variation in grain size and film thickness, i.e., different grain sizes for a given film thickness. As we have previously reported, encapsulation of $\mathrm{Cu}$ (as well as $\mathrm{Ru}, \mathrm{Au}$, and $\mathrm{Pt}$ ) films significantly

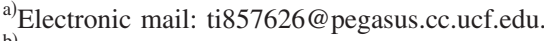

b)Electronic mail: krcoffey@mail.ucf.edu. reduces void formation upon annealing. ${ }^{10}$ Moreover, identical interfaces on the top and bottom of the $\mathrm{Cu}$ film provide indistinguishable scattering conditions; as a result, the effect of surface scattering can be easily studied. Transmission electron microscopy (TEM) based techniques are used extensively to characterize the thin films, including hand tracing of grain boundaries to provide average grain sizes from samples of 412 to 1,563 grains. This independent variation and quantification of film thickness and grain size allows the contribution of grain boundary scattering to the classical size effect to be clearly observed and found to be significantly greater than that of the surface scattering at the $\mathrm{Cu} / \mathrm{SiO}_{2}$ interfaces.

\section{EXPERIMENT}

$\mathrm{SiO}_{2}$-encapsulated $\mathrm{Cu}$ thin films were prepared on $\mathrm{Si}$ (100) substrates with $150 \mathrm{~nm}$ of thermally grown $\mathrm{SiO}_{2}$ $\left(1100{ }^{\circ} \mathrm{C}\right.$ in dry $\left.\mathrm{O}_{2}\right)$ to provide a homogenous surface for later deposition and to ease the subsequent preparation of samples for transmission electron microscopy (TEM). Prior to film deposition, the substrates were RF sputter cleaned and cooled to $-40{ }^{\circ} \mathrm{C}$ by contact with a liquid nitrogen cooled $\mathrm{Cu}$ plate. An underlayer of $20 \mathrm{~nm}$ of $\mathrm{SiO}_{2}$ was sputter deposited prior to the $\mathrm{Cu}$ film deposition and a $20 \mathrm{~nm} \mathrm{SiO}$ overlayer was subsequently similarly deposited to form a $\mathrm{SiO}_{2} / \mathrm{Cu} / \mathrm{SiO}_{2}$ structure. The $\mathrm{Cu}$ layer was deposited by DC sputter deposition from high purity $(99.9999 \%) \mathrm{Cu}$ targets at a rate of $0.6 \mathrm{~nm} / \mathrm{s}$ onto the electrically grounded substrate. All depositions were performed in 4 mTorr of Ar. A deposition chamber pressure in the $10^{-9}$ Torr range was obtained prior to film deposition and the nominally $99.999 \%$ purity $\mathrm{Ar}$ 


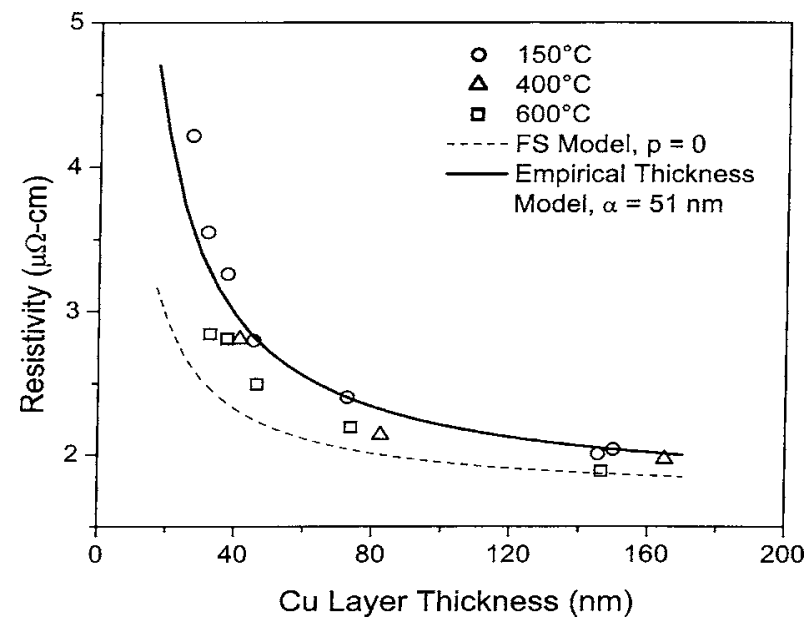

FIG. 1. The resistivity of $\mathrm{SiO}_{2} / \mathrm{Cu} / \mathrm{SiO}_{2}$ thin films annealed at 150,400 , and $600{ }^{\circ} \mathrm{C}$ as a function of $\mathrm{Cu}$ layer thickness. The data points correspond to samples A-O in Table I. The dotted curve is the resistivity prediction of the Fuchs-Sondheimer (FS) model for maximum surface scattering with specular scattering factor $p$ equal to zero. The solid curve is the resistivity prediction of the empirical thickness model with $\alpha=51 \mathrm{~nm}$. Both models are described in Table II.

was passed through a hot reactive metal getter purifier (SAES Pure Gas) prior to introduction to the chamber. Film thicknesses were determined by x-ray reflectivity. ${ }^{11}$ Samples A-G from Table I were annealed at $150{ }^{\circ} \mathrm{C}$, and samples $\mathrm{K}-\mathrm{O}$ were annealed at $600{ }^{\circ} \mathrm{C}$ for $30 \mathrm{~min}$ in a tube furnace. Samples H-J were annealed at $400{ }^{\circ} \mathrm{C}$ for $6 \mathrm{~s}$ using rapid thermal annealing. For both furnace types, the use of a reducing process gas $\left(\mathrm{Ar}+3 \% \mathrm{H}_{2}\right.$ or $\mathrm{Ar}+5 \% \mathrm{H}_{2}$ instead of $100 \% \mathrm{Ar}$ ) at $1 \mathrm{~atm}$ was found to be necessary to avoid formation of $\mathrm{Cu}$ oxides within the sample. Annealing at the lowest temperature of $150{ }^{\circ} \mathrm{C}$ for $30 \mathrm{~min}$ was used to prevent the decrease of sample resistivity with storage time as a result of room-temperature recrystallization and grain growth, ${ }^{12}$ and to prevent grain growth during TEM sample preparation. Annealing treatments at $400{ }^{\circ} \mathrm{C}$ for $6 \mathrm{~s}$ and at $600{ }^{\circ} \mathrm{C}$ for $40 \mathrm{~min}$ were used to provide increased grain sizes.

Plan-view TEM samples were prepared from pieces of all the samples described in Table I using a back-etching technique, thinning initially with $\mathrm{HF}+\mathrm{HNO}_{3}$ solution and subsequently a diluted HF solution. The microscopy was performed in a Tecnai F30 microscope operating at $300 \mathrm{kV}$. The samples were examined by high angle annular dark field (HAADF) imaging in STEM mode at relatively low magnifications to assess the fraction of voids (pinholes) present in the film. The samples were also examined by hollow cone dark field (HCDF) imaging in TEM mode to provide the highest diffraction contrast for grain size measurements. An example of an HCDF image with inverted contrast is given in Fig. 2. Multiple images of each field of view were acquired at different sample tilts to provide variation in the diffraction contrast. All grain boundaries in enlarged prints of the tilt series were hand-traced onto transparency sheets using a fine point permanent marker. The twin boundaries within grains were excluded. This hand tracing is necessary because the complex contrast in TEM images, including HCDF images, precludes the use of automated image analysis methods. The black-on-clear hand tracings were analyzed using image $\mathrm{J}^{13}$ The reported grain size is the diameter of a circle with an area equal to the average of the grain areas. The statistical errors on the mean grain size are determined from the size of the data set, assuming the grain size distribution to be lognormal. The errors shown are quoted as $2 \sigma$ values at a 95\% confidence level for the given grain population. $^{14}$

The sheet resistance of the samples was measured by a commercial four-point-contact probe system (Signatone QUADPRO). The four contact probes readily broke through the $\mathrm{SiO}_{2}$ overlayer to make electrical contact to the $\mathrm{Cu}$ film below. Thus, the measurement of the resistivity of the encap-

TABLE I. Annealing temperature and void area percent, thickness, resistivity, and grain size data for the $\mathrm{SiO}_{2}$-encapsulated $\mathrm{Cu}$ thin films A through $\mathrm{O}$.

\begin{tabular}{lccccrr}
\hline \hline Sample & $\begin{array}{c}\text { Anneal } \\
\left({ }^{\circ} \mathrm{C}\right)\end{array}$ & $\begin{array}{c}\text { Voiding } \\
(\%)\end{array}$ & $\begin{array}{c}\text { Thickness } \\
(\mathrm{nm})\end{array}$ & $\begin{array}{c}\rho @ 398 \mathrm{~K} \\
(\mu \Omega \mathrm{cm})\end{array}$ & $\begin{array}{c}\text { Grain dia. } \\
(\mathrm{nm})\end{array}$ & $\begin{array}{c}\text { Grains } \\
\text { Measured }\end{array}$ \\
\hline $\mathrm{A}$ & 150 & 0.2 & 27.1 & 4.22 & $41 \pm 3$ & 484 \\
$\mathrm{~B}$ & 150 & 0.1 & 31.7 & 3.55 & $41 \pm 2$ & 525 \\
$\mathrm{C}$ & 150 & 1.3 & 37.5 & 3.26 & $65 \pm 3$ & 1,362 \\
$\mathrm{D}$ & 150 & 0.0 & 45.3 & 2.80 & $101 \pm 4$ & 919 \\
$\mathrm{E}$ & 150 & 0.0 & 72.7 & 2.40 & $172 \pm 8$ & 872 \\
$\mathrm{~F}$ & 150 & 0.0 & 145.6 & 2.01 & $248 \pm 17$ & 412 \\
$\mathrm{G}$ & 150 & 0.2 & 150.0 & 2.04 & $344 \pm 20$ & 525 \\
$\mathrm{H}$ & 400 & 2.1 & 41.2 & 2.81 & $88 \pm 3$ & 1,563 \\
$\mathrm{I}$ & 400 & 1.4 & 82.4 & 2.14 & $221 \pm 11$ & 785 \\
$\mathrm{~J}$ & 400 & 0.0 & 164.8 & 1.97 & $419 \pm 22$ & 662 \\
$\mathrm{~K}$ & 600 & 2.4 & 32.6 & 2.84 & $68 \pm 4$ & 452 \\
$\mathrm{~L}$ & 600 & 1.9 & 37.5 & 2.81 & $81 \pm 4$ & 576 \\
$\mathrm{M}$ & 600 & 1.5 & 46.4 & 2.50 & $113 \pm 8$ & 419 \\
$\mathrm{~N}$ & 600 & 0.9 & 73.7 & 2.19 & $220 \pm 10$ & 1045 \\
$\mathrm{O}$ & 600 & 0.6 & 146.5 & 1.89 & $466 \pm 17$ & 1520 \\
\hline \hline
\end{tabular}




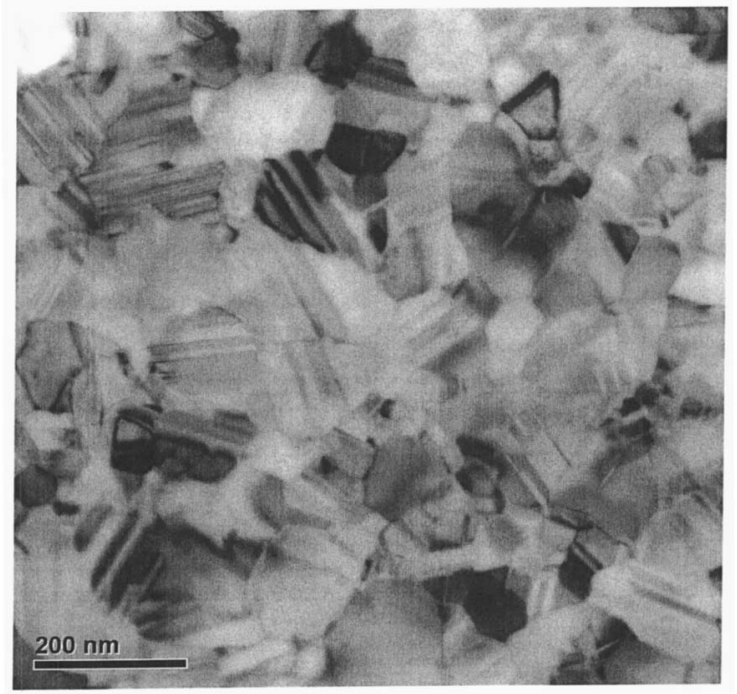

FIG. 2. An inverted contrast hollow cone dark field image of sample L as described in Table I. The image shows the complex contrast that prevents automated grain size analysis.

sulated $\mathrm{Cu}$ film was not affected by the presence of the insulating overlayer. Furthermore, since the $\mathrm{Cu}$ film sheet resistance was being measured with separate current and voltage sensing leads, the electrical resistances of the probe contact points did not affect the $\mathrm{Cu}$ film sheet resistance measurement.

The film resistivity data was compared to various models described below, and optimized values for the parameters appropriate to each model were determined by the minimization of the sum of the squared errors using the software MATHEMATICA from Wolfram Research. ${ }^{15}$ The error tolerances given for these model parameters is the deviation required to provide a $50 \%$ increase in the sum of the squared error above that of the optimized value for each parameter.

\section{RESULTS AND DISCUSSION}

Table I lists the annealing temperature, void fraction, thickness, room-temperature resistivity, grain size, and number of grains measured for the fifteen samples (A-O) studied. The resistivities of all the samples compare well with those for $\mathrm{Cu}$ films with similar thicknesses reported elsewhere. 5,6

Before fitting the film resistivity results to various models, the role of impurities, surface roughness, and film voiding in film resistivity will be discussed. Impurity scattering due to contamination is a potential concern for thin films. However, the comparatively low resistivity of the samples and the care taken in processing (for example, the absence of a resistivity increase due to interdiffusion with increasing anneal temperature) suggest that a uniform purity for the films can be assumed.

The contributions from voids and from film surface roughness to resistivity are found to be small. In detail, the rms roughness of the upper $\mathrm{SiO}_{2}$ surface of the samples, as measured by atomic force microscopy, ranges from 0.9 to $1.3 \mathrm{~nm}$, generally increasing with sample thickness.
These low roughness values are presumably due to the low temperature of $\mathrm{Cu}$ deposition and encapsulation. The work of Rossnagel and Kuan ${ }^{5}$ suggests that this range of roughness should result in small resistivity variations $(0.04 \mu \Omega \mathrm{cm})$, justifying the neglect of this factor in our analysis. Similarly, the area fraction of voids in the samples ranges from only $0.0 \%$ to $2.1 \%$. A simple geometric model for the redistribution of $\mathrm{Cu}$ with voiding indicates that sheet resistance errors less than $1 \%$ are to be expected for void fractions of $4 \%$ and less. Since this contribution to overall resistivity is small, it will also be neglected.

Given the small contributions of surface roughness and film voiding to film resistivity, the analysis of sample resistivities will focus on film thickness and film grain size effects only. In doing so, the contributions from other microstructural features such as defects in grain interiors, as well as twin boundaries, ${ }^{16}$ are neglected. Furthermore, and for the time being, all grain boundaries will be treated equal, and differences in scattering from high and low angle grain boundaries will be ignored. In the future, when methodologies for easy identification of twins and mapping of grain boundary character distribution in nanograined films are developed, more detailed models of resistivity-microstructure effects can be considered.

Table II lists a series of existing models and an empirical model for resistivity as a function of thickness $d$ and/or grain size $g$. For all models, a common value for the "bulk" or intrinsic resistivity $\left(\rho_{i}\right)$ of the thin films samples of $1.7 \mu \Omega \mathrm{cm}$ is used. ${ }^{17}$ Each model has its own additional variable parameter(s) and the table includes the equation defining the model, the optimum value(s) for its parameters, and the sum of the square error when minimized, to serve as an indication of the ability of the model to correctly describe the experimental data.

The oldest (1938) and most widely accepted model for the classical size effect is that of Fuchs-Sondheimer ${ }^{3}$ (FS) and is based upon the assumption of a fixed probability $p$ of electrons specularly scattered from the conductor surfaces. These specularly scattered electrons do not contribute to a resistivity increase, whereas the diffusely scattered electrons (with probability $1-p$ ) result in an increasing resistivity for thinner conductors. Figure 1 includes a plot of this model with $p$ $=0$, maximizing the model's resistivity increase. As can be seen in the figure, while this model is qualitatively correct, in that the predicted film resistivity increases with decreasing film thickness, it is unable to correctly predict the extent of resistivity increase and provides the highest summed square error of $5.71 \mu \Omega^{2} \mathrm{~cm}^{2}$.

An empirical thickness model is also shown in Fig. 1, having the same inverse thickness dependence as the FS model, but a greater value for the coefficient of resistivity increase, $\alpha$. This model provides an improved agreement with experiment and a reduced summed square error of $0.95 \mu \Omega^{2} \mathrm{~cm}^{2}$. The optimal $\alpha$ value $(51 \mathrm{~nm})$ for this model corresponds to a specular scattering fraction, $p=-1.05$, i.e., a 
TABLE II. Analytical expressions for the Fuchs-Sondheimer (FS) (Ref. 3), Mayadas-Shatzkes (MS) (Ref. 4), a combined MS and FS (MS, FS) (Ref. 5), and an empirical thickness model for the resistivity of classical size effect are shown, where $d$ is $\mathrm{Cu}$ film thickness, $g$ is $\mathrm{Cu}$ mean grain size, $\rho_{i}$ is the intrinsic resistivity, and $l$ is the electronic mean free path of the $\mathrm{Cu}$ thin film samples. The optimized model parameters for samples A-O with the sum of the residual squared error for each model is also given, where $p$ is the surface secularity coefficient, $R$ is the grain boundary reflection-coefficient, and $\alpha$ is an empirical parameter.

\begin{tabular}{|c|c|c|c|}
\hline Model & Model description & Sum square error & Optimized parameters \\
\hline FS & $\rho_{\mathrm{FS}}=\rho_{\mathrm{i}}\left(1+\left(\frac{\lambda}{\mathrm{d}}\right)(1-\mathrm{p})\left(\frac{3}{8}\right)\right)$ & 5.71 & $p=0$ \\
\hline Empirical thickness & $\rho_{\mathrm{ET}}=\rho_{\mathrm{i}}\left(1+\frac{\alpha}{\mathrm{d}}\right)$ & 0.95 & $\alpha=51 \pm 8$ \\
\hline MS & $\begin{array}{c}\rho_{\mathrm{MS}}=\rho_{\mathrm{i}}\left\{3\left(\frac{1}{3}-\left(\frac{1}{2}\right)\left(\frac{\lambda}{\mathrm{g}}\right)\left(\frac{R}{1-R}\right)+\left(\frac{\mathrm{R}}{1-\mathrm{R}} \frac{\lambda}{\mathrm{g}}\right)^{2}-\left(\frac{\mathrm{R}}{1-\mathrm{R}} \frac{\lambda}{\mathrm{g}}\right)^{3}\right.\right. \\
\left.\left.\times \ln \left[1+\frac{1-\mathrm{R}}{\mathrm{R}} \frac{\mathrm{g}}{\lambda}\right]\right)\right\}^{-1}\end{array}$ & 0.37 & $R=0.50 \pm 0.03$ \\
\hline MS-FS & $\begin{array}{c}\rho_{\mathrm{MS} \cdot \mathrm{FS}}=\rho_{\mathrm{i}}\left\{\frac{3}{8}(1-\mathrm{P}) \frac{\lambda}{\mathrm{d}}+\left(3\left(\frac{1}{3}-\left(\frac{1}{2}\right)\left(\frac{\lambda}{\mathrm{g}}\right)\left(\frac{R}{1-R}\right)+\left(\frac{\mathrm{R}}{1-\mathrm{R}} \frac{\lambda}{\mathrm{g}}\right)^{2}\right.\right.\right. \\
\left.\left.\left.-\left(\frac{\mathrm{R}}{1-\mathrm{R}} \frac{\lambda}{\mathrm{g}}\right)^{3} \ln \left[1+\frac{1-\mathrm{R}}{\mathrm{R}} \frac{\mathrm{g}}{\lambda}\right]\right)\right)^{-1}\right\}\end{array}$ & 0.34 & $\begin{array}{c}p=0.60 \pm 0.2 \\
R=0.45 \pm 0.03\end{array}$ \\
\hline
\end{tabular}

value of $p<0$, within the context of the FS model, which clearly has no physical meaning despite a better fit to the experimental results.

An alternative view of the classical size effect to that of Fuchs and Sondheimer was proposed by Mayadas and Shatzkes in 1970. ${ }^{4}$ Rather than surface scattering, the MayadasShatzkes (MS) model is based upon the transmission or reflection of electrons at the grain boundaries within the polycrystalline sample. This model assumes that all grain boundaries are either parallel or perpendicular to the direction of current flow, and further assumes the specular reflection of electrons from the exterior surfaces and from the grain boundaries parallel to the direction of current flow. Electrons incident on the grain boundaries perpendicular to the direction of current flow are presumed to be reflected (with probability $R$ ) or transmitted (with probability $1-R$ ). Figure 3 compares the MS model with $R=0.50$ with the experimental data of Table I, and the visual perception of an improved fit for this model is reflected in the dramatic reduction of the summed square error to $0.37 \mu \Omega^{2} \mathrm{~cm}^{2}$.

Although the resistivity of the samples can be better described as a function of grain size alone (Fig. 3, Table II), than as a function of thickness alone (Fig. 1, Table II), further assessment of the relative contributions of surface and grain boundary scattering is warranted. In doing so, a combined Mayadas-Shatzkes and Fuchs-Sondheimer (MS-FS) model is used wherein Matthiessen's rule is presumed valid and these two resistivity increases are simply summed. ${ }^{7,9}$ The MS-FS model provides a small further reduction in the summed square error (as expected for an additional degree of freedom) to $0.34 \mu \Omega^{2} \mathrm{~cm}^{2}$ and yields a specular surface scattering coefficient $p$ equal to 0.6 and a grain boundary reflection coefficient $R$ equal to 0.45 . The reflection coefficient of 0.45 is higher than previous reports, ${ }^{7,9,18}$ but it should be noted that these works had the two shortcomings noted above: (i) they lacked samples having an independent variation of grain size and thickness, and thus lacked the necessary degree of freedom to make a relative assessment, and (ii) the grain size of the samples was either not measured or measured only for a small set of grains. The relative magnitudes of the grain boundary and the surface scattering terms for the MS-FS model are shown in Fig. 4, where the horizontal axis represents both the grain size and the film thickness. As can be seen, the contribution of grain boundary scattering to the resistivity increase is clearly greater than that for the surface scattering and becomes increasingly dominant as grain size/film thickness decreases.

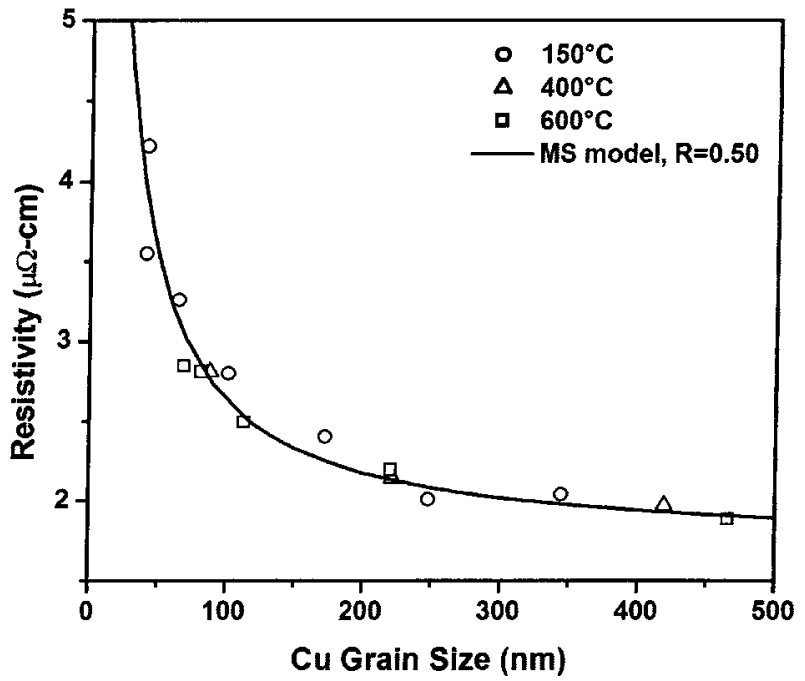

FIG. 3. The resistivity of $\mathrm{SiO}_{2} / \mathrm{Cu} / \mathrm{SiO}_{2}$ thin films annealed at 150,400 , and $600{ }^{\circ} \mathrm{C}$ as a function of $\mathrm{Cu}$ grain size. The data points correspond to samples A-O in Table I. The solid curve is the resistivity prediction of the Mayadas-Shatzkes (MS) model using the reflection coefficient $R=0.5$, as shown in Table II. 


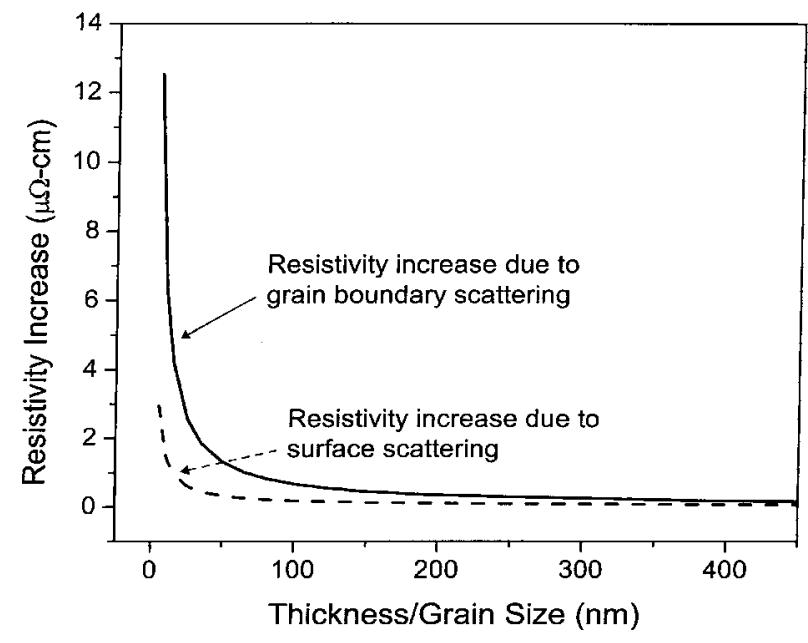

FIG. 4. The solid and dashed curves are the individual resistivity contributions of the MS and FS terms, respectively, for the combined MS-FS model of grain boundary and surface scattering using $p=0.6$ and $R=0.45$, as described in Table II.

While the MS model provides a good fit and can be considered to be an adequate description of our data, there is a residual error of 0.37 and deviations of the experimental data points from the model curve that can be seen in Fig. 3. This residual error is only marginally reduced (to 0.34 ) by inclusion of FS scattering, and thus the source of the remaining error needs to be understood as either experimental variations in our data and/or as consequences of unrecorded sample-to-sample variations that are neglected in the course of the derivations of the models used. An example of the latter is the neglect of grain boundary character in the derivation of the MS model. While experimentally we can determine the grain size and hence the density of grain boundaries in the sample, we are not characterizing the grain boundaries as to the degree of disorder or misalignment present, and are simply assuming that all grain boundaries scatter equally. This is not strictly true, and, in fact, we intuitively expect general high-angle grain boundaries to scatter more than low-angle or special grain boundaries (e.g., coherent twin boundaries). However, it is reasonable to assume similar "average" grain boundary scattering amongst our samples, but variations in this "average" grain boundary character will be responsible for resistivity variations among samples and residual error in the modeling of that resistivity.

With regard to semiconductor interconnects, conductor lines will have greater surface scattering than the blanket thin films described here owing to the presence of additional surfaces. However, for lines having a height that is five times the linewidth, as is typical, only a $20 \%$ increase in surface scattering is expected. Thus, we expect the partitioning of surface and grain boundary scattering to be similar for lines and films having grain sizes, separation between surfaces (linewidth), and $\mathrm{Cu} / \mathrm{SiO}_{2}$ interfaces similar to those studied here. However, it should be noted that $\mathrm{Cu}$ interconnects do not commonly have the $\mathrm{Cu} / \mathrm{SiO}_{2}$ interfaces studied here, but rather the need for diffusion barriers/adhesion layers for $\mathrm{Cu}$ interconnects results in the interposition of a (typically) high resistivity metal, namely, Ta on an underlayer of TaN, between the $\mathrm{Cu}$ and the dielectric, and this may change the surface scattering contribution.

\section{CONCLUSIONS}

$\mathrm{SiO}_{2}$-encapsulated $\mathrm{Cu}$ film samples, with independent variation of grain size and thickness were prepared, and their grain sizes and pinhole densities were measured in the electron microscope. Together with film resistivity measurements, this experimental methodology allows a quantitative partitioning of grain boundary and surface scattering to be carried out. The significance of grain size to sample resistivity is clearly demonstrated. Despite its historical acceptance, the independent significance of film thickness to resistivity is not clearly demonstrated in these data, providing only a small improvement in model error performance. When both effects are considered simultaneously, it is shown that, for equal grain size and film thickness, the resistivity increase due to grain boundary scattering is significantly greater than that for surface scattering.

\section{ACKNOWLEDGMENTS}

We gratefully acknowledge the financial support of the Semiconductor Research Corporation, Task 1292.008, and our many discussions with colleagues. Partial support from the MRSEC program of the NSF under DMR-0520425 is also gratefully acknowledged. Bede Scientific, Inc. is thanked for their assistance with the analysis of our $\mathrm{x}$-ray reflectivity data.

${ }^{1}$ J. J. Thomson, Proc. Cambridge Philos. Soc. 11, 120 (1901).

${ }^{2}$ F. Chen and D. Gardner, IEEE Electron Device Lett. 19, 508 (1998).

${ }^{3}$ K. Fuchs, Proc. Cambridge Philos. Soc. 34, 100 (1938); E. H. Sondheimer, Adv. Phys. 1, 1 (1952).

${ }^{4}$ A. F. Mayadas and M. Shatzkes, Phys. Rev. B 1, 1382 (1970).

${ }^{5}$ S. M. Rossnagel and T. S. Kuan, J. Vac. Sci. Technol. B 22, 240 (2004).

${ }^{6}$ W. Zhang, S. H. Brongersma, T. Clarysse, V. Terzieva, E. Rosseel, W. Vandervorst, and K. Maex,J. Vac. Sci. Technol. B 22, 1830 (2004) .

${ }^{7}$ J. J. Plombon, E. Andideh, V. M. Dubin, and J. Maiz, Appl. Phys. Lett. 89, 113124 (2006).

${ }^{8}$ D. Josell, C. Burkhard, Y. Li, Y. W. Cheng, R. R. Keller, C. A. Witt, D. R. Kelley, J. E. Bonevich, B. C. Baker, and T. P. Moffat, J. Appl. Phys. 96, 759 (2004).

${ }^{9}$ W. Steinhögl, G. Schindler, G. Steinlesberger, M. Traving, and M. Engelhardt, J. Appl. Phys. 97, 023706 (2005).

${ }^{10}$ P. R. Gadkari, A. P. Warren, R. M. Todi, R. V. Petrova, and K. R. Coffey, J. Vac. Sci. Technol. A 23, 1152 (2005).

${ }^{11}$ M. Worminton, C. Panaccione, K. M. Matney, and D. K. Bowen, Philos. Trans. R. Soc. London, Ser. A 357, 2827 (1999).

${ }^{12}$ J. M. E. Harper and K. P. Rodbell, J. Vac. Sci. Technol. B 15, 763 (1997).

${ }^{13}$ http://rsb.info.nih.gov/ij/

${ }^{14}$ D. T. Carpenter, J. M. Rickman, and K. Barmak, J. Appl. Phys. 84, 5843 (1998).

${ }^{15}$ MATHEMATICA, Version 5, Wolfram Research (www.wolfram.com).

${ }^{16}$ L. Lu, Y. Shen, X. Chen, L. Qian, and K. Lu, Science 304, 422 (2004).

${ }^{17}$ D. R. Lide, CRC Handbook of Chemistry and Physics, 75th ed. (CRC, Boca Raton, FL, 1994), pp. 12-41.

${ }^{18}$ G. B. Alers, J. Sukamio, S. Park, and G. Harm, Semicond. Int. 29, 38 (2006). 